TRANSACTIONS OF THE

AMERICAN MATHEMATICAL SOCIETY

Volume 354, Number 10, Pages 3927-3954

S 0002-9947(02)03022-2

Article electronically published on June 10, 2002

\title{
ON THE CROSSING NUMBER OF POSITIVE KNOTS AND BRAIDS AND BRAID INDEX CRITERIA OF JONES AND MORTON-WILLIAMS-FRANKS
}

\author{
A. STOIMENOW
}

\begin{abstract}
We give examples of knots with some unusual properties of the crossing number of positive diagrams or strand number of positive braid representations. In particular, we show that positive braid knots may not have positive minimal (strand number) braid representations, giving a counterpart to results of Franks-Williams and Murasugi. Other examples answer questions of Cromwell on homogeneous and (partially) of Adams on almost alternating knots.

We give a counterexample to, and a corrected version of, a theorem of Jones on the Alexander polynomial of 4-braid knots. We also give an example of a knot on which all previously applied braid index criteria fail to estimate sharply (from below) the braid index. A relation between (generalizations of) such examples and a conjecture of Jones that a minimal braid representation has unique writhe is discussed.

Finally, we give a counterexample to Morton's conjecture relating the genus and degree of the skein polynomial.
\end{abstract}

\section{INTRODUCTION}

The braid index $b(L)$ of a knot or link $L$ is defined to be the minimal number of strands of a braid whose closure is the link. (That such a braid always exists was first shown by Alexander [3.) To determine the braid index of $L$, one seeks general lower and upper estimates on $b(L)$. Upper estimates can be obtained in the obvious way by writing down braid representations of $L$ (although finding a braid representation realizing $b(L)$ may sometimes be difficult); so the harder problem is to estimate $b(L)$ from below. Very little was known in general on this problem (except some early results of Murasugi [45] for 3-braids, which required much effort), until Jones discovered his polynomial invariant in 1984 [27]. His construction made heavy use of braid representations, and thus he obtained several conditions for knots, in particular of low braid index. Shortly after that the HOMFLY (skein) polynomial [24, 32] was discovered, which gave rise to what is now the most commonly used braid index estimate 11 for $b(L)$, the MFW inequality [34, 21]. This inequality determined the braid index of all knots in [48], except five. They were

Received by the editors November 10, 2001 and, in revised form, February 12, 2002.

2000 Mathematics Subject Classification. Primary 57M25; Secondary 20F10, 20 F36.

Supported by a DFG postdoc grant.

${ }^{1}$ In the sequel we will be interested only in lower estimates. 
dealt with by the 3-braid formula of Murakami [42, corollary 10.5], or by applying the MFW inequality on their 2-cable [41].

One of the central points of this paper is the study of these braid index inequalities with particular regard to positive braids.

We discuss a conjecture of Jones in 28, that a minimal braid representation has unique writhe, and relate this conjecture to the MFW inequality and its cabled versions [41]. A consequence of this relation is that on a counterexample to Jones's conjecture any cable version of the MFW inequality will fail to estimate the braid index sharply. Therefore, at the present state of the art it is very unlikely to find (that is, to prove some link to be) a counterexample to Jones's conjecture, except possibly if it is a 4-braid. 2 On the quest for such an example we found knots for which both the MFW inequality and its 2-cable version (and hence any previously applied method) fail to estimate the braid index sharply. We will show one of these knots.

Also, we consider one of Jones's original criteria in [27. We provide a counterexample to it, showing that it needs correction, and we give the corrected version.

Then we turn to Jones's unity root criteria for the Jones polynomial. We give an example showing that these criteria sometimes can estimate the braid index better than the MFW inequality, and thus deserve (although apparently neglected after $\mathrm{MFW})$ to be considered in their own right.

Another aim of the paper, which we will begin with, is to show some examples of positive knots with unusual behaviour of classical invariants as braid index and crossing number in positive braid representations and diagrams.

Positive knots are defined to be knots with diagrams of all crossings positive (see, e.g., [17]). This class of knots contains as a subclass the braid positive knots, i.e., those which are closures of positive braids 3 Such knots were studied in knot theory, inter alia because of their relevance to the theory of singularities [23] and dynamical systems [9]. Thus they have received much attention in previous publications [11] 15, 49.

Positive and braid positive knots have been studied in many papers jointly with alternating (braid) knots as a subclass of the homogeneous knots and braids 14 52. It is now known (see, e.g., 43, 44]) that reduced alternating diagrams are of minimal crossing number and that reduced alternating braid representations are of minimal strand number. Simple examples show that neither of these is true for positive/homogeneous diagrams/braid representations, so that the reasonable question is whether there always exists at least some such minimal diagram/braid representation. A partial positive answer in the case of positive braids was given in 21] for positive braids containing a full twist. In [43] it was remarked that a positive/homogeneous braid representation of minimal strand number also has minimal crossing number. (Thus the positive answer for braid representations for a given link implies a positive answer for diagrams for this link.) Here we show that the answers to both questions are in general negative.

Theorem 1. There exist knots with positive/homogeneous diagrams but with no positive/homogeneous diagrams of minimal crossing number, or with positive/

\footnotetext{
${ }^{2}$ It has been claimed by Birman [7] that the truth of this conjecture for 4-braids follows from Jones's work [28], but this claim is possibly incorrect; see $\$ 4.3$.

${ }^{3}$ Some authors very confusingly call 'positive knots' what we will call here 'braid positive knots'.
} 
homogeneous braid representations, but with no positive/homogeneous braid representations of minimal strand number.

Beside these examples, we will prove some relations between the crossing number and genus of braid positive knots. These inequalities will enable us to show that certain knots, like Perko's knot $10_{161}$, have no positive braid representations, or that the reduced positive braid representation of some others, like the closed 4braid $\left(\sigma_{1} \sigma_{3} \sigma_{2}^{2}\right)^{3} \sigma_{2}$, is unique.

In the final section, we will give examples settling two conjectures on possible inequalities between the genus and the degrees of the skein polynomial, one of which is a 15-year-old problem of Morton [37].

Most of the examples presented below were found by examining the tables of the program KnotScape of Hoste and Thistlethwaite [26]. Beside providing access to these tables, the program makes it possible to calculate their polynomial invariants and to identify a knot in the table from a given diagram. These features were used to a large extent in the calculations described below.

1.1. Definitions and notation. The $n$-strand braid group $B_{n}$ is generated by the elementary (Artin) braids $\sigma_{i}$ for $1 \leq i \leq n-1$ with relations $\sigma_{i} \sigma_{i+1} \sigma_{i}=\sigma_{i+1} \sigma_{i} \sigma_{i+1}$, called henceforth the Yang-Baxter (YB) relation, and $\left[\sigma_{i}, \sigma_{j}\right]=1$ for $|i-j|>1$ (the brackets denoting the commutator), called the commutativity relation.

By $\hat{\alpha}$ we denote the link which is the braid closure of $\alpha$. Markov's theorem (see, e.g., 36] ) says that when $\hat{\alpha}=\hat{\alpha}^{\prime}$ then $\alpha$ and $\alpha^{\prime}$ can be transformed into each other by a sequence of conjugacies in the $B_{n}$ 's and moves of the type $\alpha \leftrightarrow \alpha \sigma_{n}^{ \pm 1} \in B_{n+1}$ for $\alpha \in B_{n}$. We call the ' $\rightarrow$ ' part of this move (which augments the strand number by 1 ) stabilization, and its inverse destabilization.

By $[\beta]$ we denote the exponent sum of a braid $\beta$, that is, the image of $\beta$ under the homomorphism $B_{n} \rightarrow \mathbb{Z}$ given by $\left[\sigma_{i}\right]:=1$ for any $i$. A braid word $\beta$ is called positive if its length equals $[\beta]$, and a braid $\beta$ is positive if it has a positive word representation. (As is already apparent, we will often abuse the distinction between braids and braid words, as this will cause no confusion.) By $[\beta]_{i}$ we denote the exponent sum of the generator $\sigma_{i}$ in the braid word $\beta$, which is clearly not invariant under the YB relation. That is, $[.]_{i}$ is a homomorphism of the free group in the $\sigma_{j}$ given by $\left[\sigma_{j}\right]_{i}:=\delta_{i j}$ (where $\delta$ is the Kronecker delta).

By $P_{K}$ or $P(K)$ we denote the skein polynomial of $K$ 24], and by $v$ its nonAlexander variable. The span of $P$ in $v$ means the difference between its maximal and minimal degree in this variable. These degrees are denoted by $\max \operatorname{deg}_{v} P_{K}$ and $\min \operatorname{deg}_{v} P_{K}$, respectively. The other (Alexander) variable of $P$ is denoted by $z$. By $\max \operatorname{cf}_{z} P$ we mean the maximal coefficient of $z$ in $P$ (which is a polynomial in $v$ ), i.e. the coefficient of the maximal degree of $z$ in $P$.

The braid index $b(K)$ of a knot $K$ is defined by

$$
b(K)=\min \left\{n \mid \exists \beta \in B_{n}: \hat{\beta}=K\right\} .
$$

A lower bound for the braid index is given by the inequality of Franks-Williams 21] and Morton 34]:

$$
b(K) \geq v \text {-span } P_{K} / 2+1 .
$$

The Morton-Williams-Franks inequality will be subsequently abbreviated as 'MFW inequality' or simply as 'MFW', and its right-hand side will be called the 'MFW bound' for $K$. 
Whenever we talk of a diagram being minimal, we always mean minimality with respect to the crossing number of the knot it represents. Similarly, minimality of a braid representation is meant with respect to the braid index of its closure (i.e., that the strand number of the braid realizes this braid index).

'W.l.o.g.' will abbreviate 'without loss of generality' and 'r.h.s' (resp. 'l.h.s') 'right-hand side' (resp. 'left-hand side').

A final remark on knot tables and notation is in order. It is understood that alternative work on knot tabulation to that of Hoste, Thistlethwaite and Weeks is being done by Aneziris [4. Unfortunately, it seems like every new knot tabulator chooses and insists on his own numbering convention for knots, which will (and, in fact, already did 4 ) lead to confusion in using the different knot tables. It appears most correct to stick to the convention of the first tabulator for each crossing number. We use here the convention of Rolfsen's tables 48 for $\leq 10$ crossing knots and that of $[26$. for $\geq 11$ crossing knots, which coincides with those of the first tabulators for any crossing number except 11, where the initial (complete) tables were compiled by Conway 13. We apologize for not using his numbering. An excuse is that all calculations have been performed by KnotScape, which does not yet provide a translator between its notation and that of Conway. For uniformity reasons, we will continue using this convention in subsequent papers, too.

\section{Some interesting DiAgrams of $\mathbf{1 1}_{\mathbf{5 5 0}}$}

2.1. A positive knot with no positive minimal diagram. An intuitive question on positive knots (whose affirmation in the alternating case was one of the big achievements of the Jones polynomial) is whether any positive knot has a positive minimal (crossing number) diagram (see [53]). This was known to be true in the case when the positive knot is alternating [47] or of genus at most two [54]. The following, surprisingly simple, (non-alternating genus three) example shows that this need not be true in general.
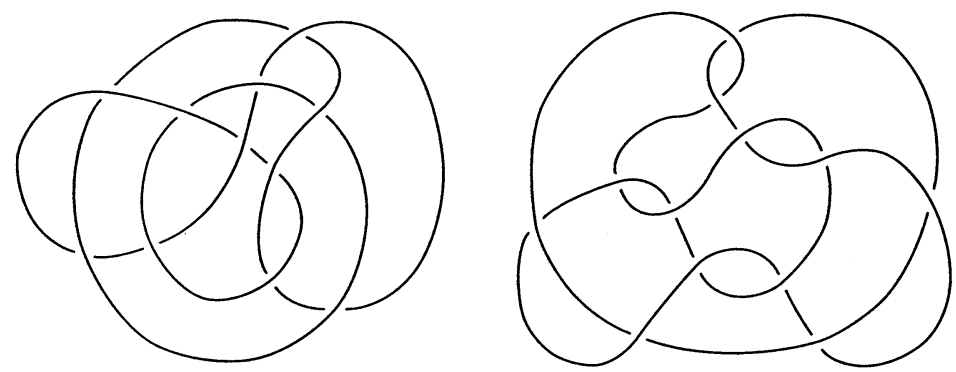

Figure 1. Two diagrams of the knot $11_{550}$. On the left is its (only) minimal diagram (which is not positive), and on the right is a positive 12 crossing diagram.

Example 1. The knot $11_{550}$ has only one 11 crossing diagram, shown on the left of figure 1. The diagram is only almost positive (i.e., it has one negative crossing). However, $11_{550}$ has a positive 12 crossing diagram, shown on the right. Thus it is

\footnotetext{
${ }^{4} \mathrm{I}$ know of at least two cases where the Hoste-Thistlethwaite numbering of the Rolfsen knots was the origin of embarrassing confusions in published material.
} 
positive, but has no positive minimal diagram. As by [14, theorem 4 and corollary 4.1] any homogeneous diagram of a positive knot must be positive, this example simultaneously provides a negative answer to question 2 in [14, §5]. (This also implies a positive answer to question 1 therein, but this answer was previously known to follow from the almost positive diagram of the Perko knot - the mirror image of the diagram of $10_{161}$ in Rolfsen's tables [48.)

Another problem for positive knots is how far the crossing number of a positive diagram can differ from the crossing number of the knot. The presently known result is obtained in [53] using the Gauß sum theory of Polyak-Viro-Fiedler.

Theorem 2 ([53]). If $D$ is a positive reduced diagram (i.e. with no nugatory crossings) of a positive knot $K$ with $c(D)$ crossings, then the crossing number $c(K)$ of $K$ satisfies $c(K) \geq \sqrt{2 c(D)}$.

The bound is clearly not very sharp, and a much better estimate appears to be true.

Conjecture 1. With the notation of theorem 2, $c(K) \geq c(D)-2 g(K)+1$, where $g(K)$ is the genus of $K$.

Here is some motivation for this conjecture.

- The conjecture is known to be true for $K$ fibered [14, corollary 5.1], of genus at most 2 [54], and for $c(D) \leq 16$ by experiment (note that only the cases where $g(K)=3$ need to be checked).

- The inequality is sharp for all positive rational knots (i.e., is the best possible) [55, and also for some other knots, e.g. $9_{16}$ (see figure 2). It is worth remarking that all knots I found so far, for which the inequality was sharp, are alternating and arborescent (this was, in particular, always the case for $g(K)=3$ and $c(D) \leq 16)$.

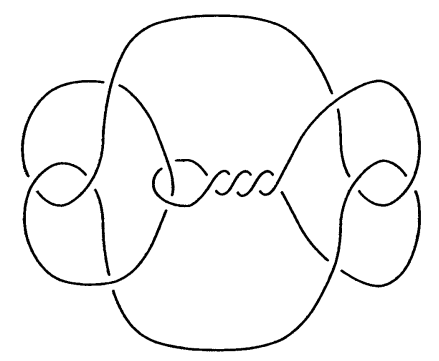

Figure 2. A positive 14 crossing diagram of the knot $9_{16}$.

A further question we can pose is

Question 1. Does a braid positive knot always have a (braid) positive minimal diagram?

We will later answer this question positively for $\leq 16$ crossing knots, but also provide evidence against it by answering negatively a question about closely related properties of braid positive knots. 
2.2. An example on almost alternating diagrams. While discussing diagrams of the knot $11_{550}$, it is worth making an aside from our positivity considerations to almost alternating diagrams.

Such diagrams were considered in [2] to be diagrams obtainable from alternating diagrams by one crossing change, and almost alternating knots are knots having such diagrams, but which are not alternating. A surprising variety of knots turns out to be almost alternating, in particular very many low crossing number knots. Using Conway's description of $\leq 11$ crossing prime knots [13] and a simple way to manipulate their Conway notation, all such non-alternating knots were found to be almost alternating with 3 exceptions. They are shown in figure 5.54 of [1], and (from left to right and in our notation) are $11_{550}, 11_{485}$ and $11_{462}$.

A computer check showed that in fact our knot $11_{550}$ is almost alternating. Two almost alternating diagrams of it are shown in figure 3 . Such diagrams show that in general it will be hard to decide on almost alternation of a given knot. No strong obstructions are known, nor is any effective method for seeking almost alternating diagrams available. (It is not hard to see, e.g., that many almost alternating knots have infinitely many almost alternating diagrams, so that an upper control on their crossing number is not possible.)

The other two knots are indeed problematic. We cannot prove them not to be almost alternating, but no almost alternating diagram was found after checking all diagrams of $\leq 16$ crossings and some diagrams of 17 crossings.
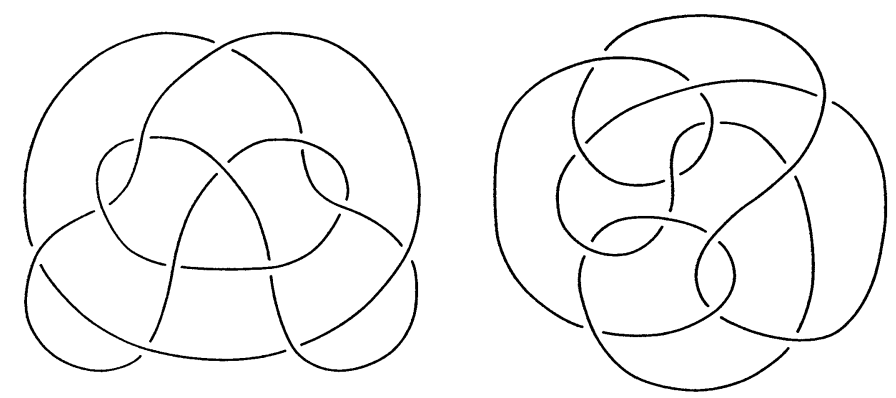

FIgURE 3 . Two almost alternating diagrams of the knot $11_{550}$.

\section{The CROSSING NUMber AND Genus of POSITIVE BRAidS}

3.1. Irreducible positive braids. In [14 corollary 5.1] it was shown that the inequality $g \geq c / 4$ holds for any positive $c$ crossing diagram of a fibered positive knot of genus $g$. This inequality in particular applies to braid positive diagrams. We will improve the inequality by showing that for braid positive knots $K$ we have $g(K) \geq c(K) / 4+2$ with finitely many exceptions. This is based on a continuation of the investigation on the crossing number of irreducible positive braids which was initiated in [53].

Definition 1. For $n \geq 2$ define the number $d_{n}$ to be the minimal number of crossings of a positive $n$-strand braid ( $n$-braid, for short) $\beta$ such that there is no positive braid $\beta^{\prime}$ of smaller crossing number, or (because of the genus) equivalently, strand number, with $\hat{\beta}=\hat{\beta}^{\prime}$, and $\hat{\beta}$ a prime knot. We call such a braid $\beta$ irreducible. 
Remark 1. In the definition no requirement is made on (the crossing number $[\beta]$ of) $\beta$ to realize $c(\hat{\beta})$. Anything that follows would remain true with this modified definition, but definition 1 appears more natural because the answer to question 1 is unclear.

Proposition 1. The values of $d_{n}$ for small $n$ are as follows:

\begin{tabular}{c|c|c|c|c|c}
$n$ & 2 & 3 & 4 & 5 & 6 \\
\hline$d_{n}$ & 3 & 8 & 11 & 16 & 19
\end{tabular}

We will later describe how they were obtained.

Here we show the following theorem.

\section{Theorem 3.}

1) For $n \geq 7$ we have $d_{n} \geq 2 n+6$.

2) $d_{n} \leq \frac{9}{2} n-\left\{\begin{array}{ll}3 & n \equiv 0(4) \\ \frac{9}{2} & n \equiv 1(4) \\ 6 & n \equiv 2(4) \\ \frac{3}{2} & n \equiv 3(4)\end{array}=\frac{9}{2} n-\frac{3}{2}((n+1) \bmod 4+1)\right.$.

Proof. 1) Take a braid $\beta$ realizing $d_{n}$ for $n$ fixed. For irreducibility reasons we must have $[\beta]_{i} \geq 2$ for any $1 \leq i \leq n-1$. Our aim is to show that

$$
\sum_{i=1}^{3}[\beta]_{n-i} \geq 10 \text { and similarly } \sum_{i=1}^{3}[\beta]_{i} \geq 10 .
$$

Then $[\beta] \geq 2 n+6$ for $3<n-3$, i.e. $n \geq 7$.

When writing $\beta=\prod_{j} \sigma_{i_{j}}$, we can assume modulo $\mathrm{YB}$ relations that the index $\operatorname{sum} \sum_{j} i_{j}$ is minimal. Using this word representation for $\beta$, consider the subword of $\beta$ made up of $\sigma_{n-2}$ and $\sigma_{n-1}$, keeping separate parts separated by subwords of $\beta$ made up of $\sigma_{n-i}, i>2$. Thus we can write $\beta$ as

$$
\begin{aligned}
& \left(\sigma_{n-2}^{a_{1,1}} \alpha_{1,1} \sigma_{n-2}^{a_{1,2}} \alpha_{1,2} \ldots \sigma_{n-2}^{a_{1, n}} \alpha_{1, n_{1}} \sigma_{n-1}^{b_{1}}\right) \\
& \quad \ldots\left(\sigma_{n-2}^{a_{k, 1}} \alpha_{k, 1} \sigma_{n-2}^{a_{k, 2}} \alpha_{k, 2} \ldots \sigma_{n-2}^{a_{k, n}} \alpha_{k, n_{k}} \sigma_{n-1}^{b_{k}}\right),
\end{aligned}
$$

with the $\alpha_{l, j}$ standing for subwords containing only $\sigma_{n-i}, i>2$.

We can use commutativity relations to ensure that each one of the subwords $\alpha_{l, j}$ contains at least one $\sigma_{n-3}$, and that all $n_{i}>0$.

We have that $k \geq 2$, else $\beta$ decomposes. If $n_{l}=1$ and $a_{l, 1}=1$ for some $l$, we can apply (after some commutativity relations) a YB relation $\sigma_{n-1} \sigma_{n-2} \sigma_{n-1} \rightarrow \sigma_{n-2} \sigma_{n-1} \sigma_{n-2}$ to reduce the index sum of the word, a contradiction to our assumption. Thus, assume that $\sum_{i=1}^{n_{l}} a_{l, i} \geq 2$ for all $1 \leq l \leq k$. Therefore,

$$
[\beta]_{n-2}=\sum_{l=1}^{k} \sum_{i=1}^{n_{l}} a_{l, i} \geq 4 .
$$

Case 1. Assume that $[\beta]_{n-2}=4$ and $[\beta]_{n-1}=2$. According to the distribution of $\sigma_{n-2}$ we have 3 possibilities.

Case 1.1. $k=2, n_{1}=n_{2}=2$ and $a_{1,1}=a_{1,2}=a_{2,1}=a_{2,2}=1$.

Case 1.2. $k=2, n_{1}=2, n_{2}=1, a_{1,1}=a_{1,2}=1$, and $a_{2,1}=2$. 
Case 1.3. $k=2, n_{1}=n_{2}=1$ and $a_{1,1}=a_{2,1}=2$.

Case 1.3 is excluded, because the closure is not connected (i.e., not a knot). In cases 1.1 and 1.2 the following argument applies.

Since all $\alpha_{i, j}$ contain the letter $\sigma_{n-3}$, we have

$$
[\beta]_{n-3} \geq \sum_{i=1}^{k} n_{i} .
$$

Thus in case 1.1 we have $[\beta]_{n-3} \geq 4$. If in case $1.2[\beta]_{n-3}=3$, then for one of $i=1,2, \alpha_{i, 1}$ contains the letter $\sigma_{n-3}$ exactly once. Then (after some commutativity relations) the subword $\sigma_{n-2}^{a_{i, 1}} \alpha_{i, 1} \sigma_{n-2}^{a_{i, 2}}$ can be made to admit a YB relation $\sigma_{n-2} \sigma_{n-3} \sigma_{n-2} \rightarrow \sigma_{n-3} \sigma_{n-2} \sigma_{n-3}$, a contradiction to our assumption. Thus $[\beta]_{n-3} \geq 4$, and (2) holds. (The second inequality therein follows analogously to the first one.)

Case 2. Otherwise, $[\beta]_{n-1}+[\beta]_{n-2} \geq 7$, so $\sum_{i=1}^{3}[\beta]_{n-i} \geq 9$. Again one needs to check that the case $\sum_{i=1}^{3}[\beta]_{n-i}=9$ cannot occur. For this one applies the same type of argument, but the case list becomes too large to be effectively handled manually, so it is safer to check the cases by computer (see $\$ 3.3$ for more details on this calculation).

2) We write down explicit positive braids with the given number of crossings. To show that they are irreducible, we use the value $\left|V\left(e^{\pi i / 3}\right)\right|$ (where $V$ is the invariant introduced in 27]) on their closure and apply proposition 14.6 of [28]. It is easy to see that this value is preserved by a 3-move, which in the context of braid words means cancelling subwords of the type $\sigma_{i}^{3}$. As all the braids we will write down become trivial after a sequence of such cancellations, their closure satisfies $\left|V\left(e^{\pi i / 3}\right)\right|=\sqrt{3}^{n-1}$, and thus the braids are irreducible (a more special type of this argument was given in corollary 15.5 of [28]). To show primeness, we use the result of [15].

Case 1. $n \equiv 0$ (4). Consider

$$
\left(\sigma_{1}^{3} \sigma_{2} \ldots \sigma_{n-3}^{3} \sigma_{n-2} \sigma_{n-1}^{3}\right)^{2} \sigma_{2} \sigma_{4} \sigma_{6} \ldots \sigma_{n-2} .
$$

Case 2. $n \equiv 1$ (4). Consider

$$
\beta=\left(\sigma_{1}^{3} \sigma_{2} \ldots \sigma_{n-2}^{3} \sigma_{n-1}\right)^{2} \sigma_{2} \sigma_{4} \sigma_{6} \ldots \sigma_{n-1} .
$$

Case 3. $n \equiv 2$ (4). Consider

$$
\left(\sigma_{1} \sigma_{2}^{3} \ldots \sigma_{n-2}^{3} \sigma_{n-1}\right)^{2} \sigma_{1} \sigma_{3} \sigma_{5} \ldots \sigma_{n-1} .
$$

Case 4. $n \equiv 3$ (4). Consider

$$
\left(\sigma_{1} \sigma_{2}^{3} \ldots \sigma_{n-3}^{3} \sigma_{n-2} \sigma_{n-1}^{3}\right)^{2} \sigma_{1}^{4} \sigma_{3} \sigma_{5} \ldots \sigma_{n-2} .
$$

It is easy to check that in all cases the closures are knots, and that the crossing numbers are as stated above.

The following straightforward consequence shows how far the inequality $g \geq c / 4$ for braid positive knots can be improved. 
Corollary 1. For almost all (i.e., all but finitely many) braid positive knots, $g \geq$ $c / 4+2$. Moreover, for any constant $C$ with $g \geq C \cdot c$ for almost all braid positive knots we have $C \leq \frac{7}{18}$.

Remark 2. Although we have not yet proved the values for $d_{n}$ in (1), the proof of 1) already shows that $d_{n} \geq 2 n+2$ for $n=5,6$.

Example 2. Theorem 3 can be used to show that some fibered positive knots, like Perko's, are not braid positive. $K=10_{161}$ has genus 3 and braid index 3 . If $K=\hat{\beta}$ with $\beta \in B_{n}$ positive and w.l.o.g. irreducible, then $[\beta] \geq \max \left(c(K), d_{n}\right)$, and

$$
g(K)=\frac{[\beta]-n+1}{2} \geq \frac{\max \left(c(K), d_{n}\right)-n+1}{2} .
$$

However, by remark 2 the r.h.s. is at least 4 for any $n \geq 3$, a contradiction. We will later see that this simple reasoning (given the numbers $d_{n}$ computed for enough small $n$ ) does not always work.

Proof of proposition 1 First one generates all braid positive $\leq 16$ crossing diagrams from the tables of 26] and identifies braid positive knots from them. This led to the values of $d_{n}$ for $n \leq 5$. For $n=6$ one needed to exclude 17 crossing braids. This was done by generating a superset of all irreducible 17 crossing braids (see $\$ 3.3$ for more details how this was done) and identifying their closures. All knots had $\leq 16$ crossings. That there is a 19 crossing irreducible 6 -braid will be shown by example later (see example 3).

Remark 3. It would be interesting to see how the sequence of $d_{n}$ continues. Sloane 51] reports on two sequences starting as in (1). One is related to [12] and the other one is made up of numbers $\equiv 0,3(8)$. It would be surprising if the answer were that simple, though.

\subsection{Examples.}

3.2.1. Minimal crossing number positive braid diagrams. Although it is desirable to push on for further results of the above type, there are many difficulties in controlling positive braid representations. We illustrate this by a series of examples.

Example 3. The inequality in 1) of theorem 3 cannot be improved by trivial means. As noted in [53], the braids $\left\{\beta_{n} \mid n\right.$ odd $\}$ with

$$
\beta_{n}=\left(\left(\sigma_{1} \sigma_{3} \ldots \sigma_{n-4} \sigma_{n-2}^{3}\right)\left(\sigma_{2}^{3} \sigma_{4} \ldots \sigma_{n-3} \sigma_{n-1}\right)\right)^{2}
$$

admit no YB relation. A computer check shows that $\beta_{5}$ is indeed irreducible (one of the examples showing $d_{5}=16$ ), while $\beta_{7}$ reduces to the 19 crossing 6 -braid

$$
\beta_{7}^{\prime}=\sigma_{1} \sigma_{2}^{3} \sigma_{4}^{2} \sigma_{3} \sigma_{5} \sigma_{4}^{3} \sigma_{1} \sigma_{3} \sigma_{5} \sigma_{2}^{3} \sigma_{3} \sigma_{4},
$$

thus showing $d_{6}=19$, and that $\beta_{9}$ also reduces to a 6 -braid, this time of 21 crossings.

Calculating the Fiedler polynomial $\Delta$ [20] (see also [35], but not [3]) of any of the stabilizations $\beta_{7}^{\prime \prime}$ of $\beta_{7}^{\prime}$ or cyclic permutations or flips $\left(\sigma_{i} \leftrightarrow \sigma_{n-i}\right)$ thereof, we had (writing just the honest polynomial part and using the notation of [32]) $\Delta\left(\beta_{7}^{\prime \prime}\right)=[0] 20305$, while $\Delta\left(\beta_{7}\right)=[0] 20206$, so $\beta_{7}$ and $\beta_{7}^{\prime \prime}$ are not conjugate. It is not clear whether $\beta_{7}$ is not conjugate to a stabilization of another positive conjugate of $\beta_{7}^{\prime}$. (There is an algorithm to list all such positive conjugates [39], but it is too complex, especially as I have no computer version of it.) Anyway, this example provides some evidence against an easy version of Markov's theorem for positive 
braids. In any case one cannot obtain every minimal positive braid representation from a given one just by YB moves, cyclic permutations and destabilization.

The following example shows that the argument in example 2 very often fails.

Example 4. The two mutant knots in figure 4, $15_{203432}$ and $15_{203777}$, are positive fibred of genus 6 , like many other 15 crossing knots, which are braid positive. The diagrams on the figure are the only 15 crossing diagrams of these knots, and they are not braid diagrams. In fact it turns out that both knots are not braid positive. To show this, it suffices to show that they have no positive braid representations of 17 crossings and 6 strands, and of 16 crossings and 5 strands. Indeed the knots did not occur in such representations (see \$3.3). This shows that just comparing genus and crossing number as in example 2 will in general not suffice to exclude braid positivity. Many more (several hundred) such examples (again of genus 6) occurred at crossing number 16.

We will come back to this example a little later when we consider mutation.

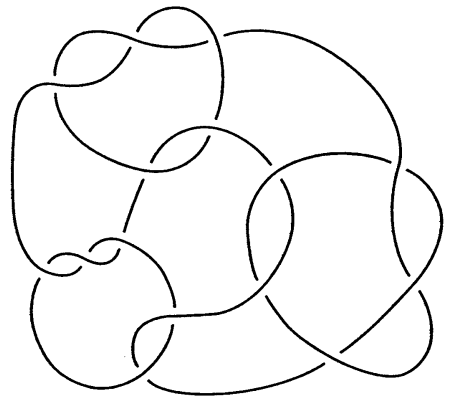

$15_{203432}$

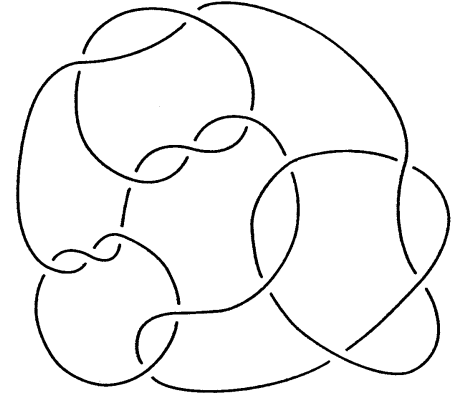

$15_{203777}$

Figure 4. Two knots which are fibered and positive, and have the same genus and crossing number as other braid positive knots, but are not braid positive.

Theorem 4. Any braid positive knot of $\leq 16$ crossings has a braid positive minimal diagram.

Proof. We proceed as follows.

First we consider crossing number $\leq 15$.

1) We identified all knots with braid positive diagrams of $\leq 15$ crossings, and found that all they have a braid positive minimal diagram.

2) Now we need to consider the cases where the braid positive knot has $\leq 15$ crossings, but all its braid positive diagrams have $>15$ crossings. We have from [14, 46] that for a braid positive $\operatorname{knot} K, \max \operatorname{deg}_{z} P_{K}=\min \operatorname{deg}_{v} P_{K}(=$ $2 g(K))$ and $\max \operatorname{cf}_{v} P_{K}=z^{\min \operatorname{deg}_{v} P_{K}}$. Thus we select all knots whose $P$ polynomial has this property. If $K$ is braid positive and $g(K)\left(=\max \operatorname{deg}_{z} P_{K} / 2\right) \leq$ 5 , then from theorem 3 and (II) we have a positive braid representation of $\leq 13$ crossings, and we checked that such knots have minimal braid positive diagrams. 
3) If $g>5$, then the knot was among those identified in 1), except the knots in example 4 .

Now consider crossing number 16.

Again we select the 16 crossing non-alternating knots satisfying the above condition on $P$ with $g \geq 6$ ( $g \leq 5$ is dealt with as above). If such knots are braid positive, then they must have braid positive diagrams of $\leq 17$ crossings (see example 4). There were 393 knots of 16 crossings with the $P$ condition for $g \geq 6$ which did not have braid positive 16 crossing diagrams. All of them had $g=6$ (i.e., no one had $g=7$ ), and none of the 17 crossing braid positive diagrams (of genus 6 ) identified to any of these knots. Thus they are all not braid positive. The same exclusion applied for the two knots in example 4 .

A final remark on minimal crossing number diagrams is that it is not true that any minimal diagram of a braid positive knot is braid positive.

Example 5. The knot $11_{444}$ is braid positive, but not all its minimal diagrams are braid positive diagrams. (This phenomenon does not occur for the braid positive knots in Rolfsen's tables.) See figure 5 .
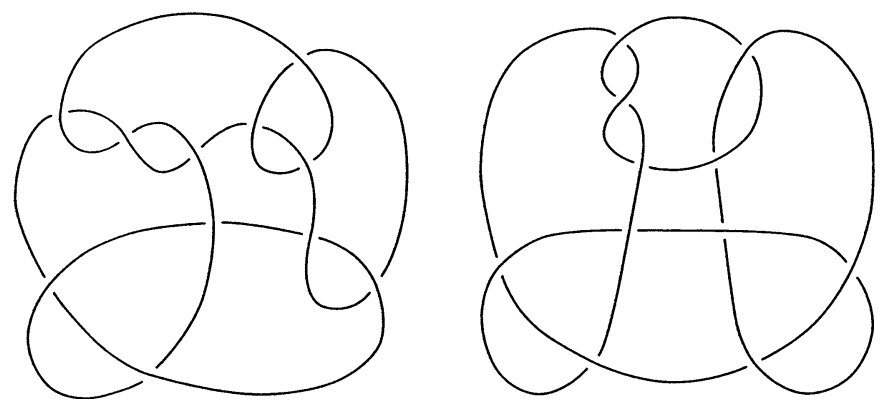

Figure 5. Two minimal diagrams of the knot $11_{444}$. On the left is a diagram which is the diagram of a closed braid, and on the right is another diagram which is not.

3.2.2. Minimal strand number positive braid representations. There is an observation of [43] relating the crossing number and braid index of braid positive knots. Murasugi showed that a minimal positive (or homogeneous) braid representation has minimal crossing number of its closure. He also showed that reduced alternating braid representations are minimal. This is trivially not true for positive braid representations (e.g. $\left(\sigma_{1} \sigma_{2}\right)^{2}$ for the trefoil), so a natural question is whether there is at least one positive minimal braid representation. In [21], it was shown that if $\beta$ factors as $\Delta^{2} \alpha$ with $\alpha$ positive and $\Delta^{2}$ being the full twist braid (generating the center of the braid group), then MFW is sharp for $\beta$, so that $\beta$ is minimal (this contains the case of torus knots considered in [43, proposition 7.5]).

However, the following examples show that both conclusions are in general problematic. The MFW inequality cannot always help to prove some positive braid representation of a given knot to be minimal, nor need such a minimal positive braid representation exist at all. 
Example 6. Among braid positive 15 crossing knots, which (except the $(2,15)$ torus knot) have genus 6 (and a positive 4-braid representation), there are two knots with MFW bound 3, see figure 6 That both knots are not closures of 3-braids can be shown using their $Q$ polynomial [10, 25] and the formula of Murakami [42] (see also [29, theorem 2]). The (only) possible value for the exponent sum of the hypothetic 3-braid representing the knot can be found from the inequalities of [34] and the degrees of $P$ in the non-Alexander variable. Thus MFW need not be sharp even on minimal positive braid representations. (Another such example will be given later in 4.4 )

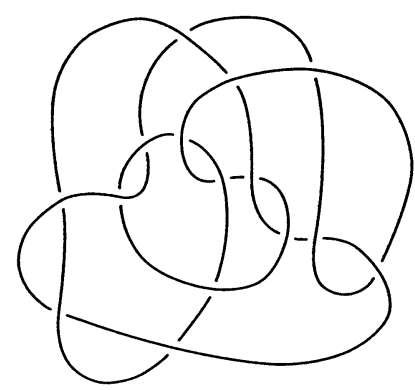

$15_{166057}$

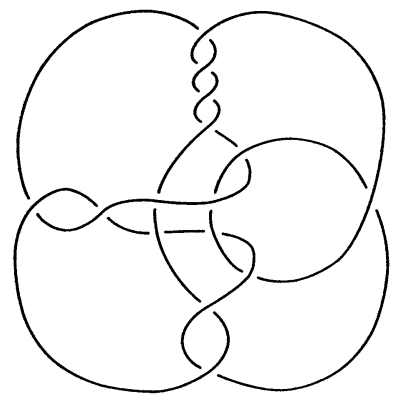

$15_{225767}$

Figure 6. Two braid positive knots with unsharp MFW.

Example 7. Considering braid positive 16 crossing knots (they are of genus 6 or 7 ), there are again two genus 6 knots (closures of positive 16 crossing 5 -braids), $16_{472381}$ and $16_{1223549}$, whose MFW bound 4 is unsharp on these braid representations (see figure 7). This time it is not clear that both knots are not of braid index 4 . The evidence against this was strengthened by their 2-cable- $P$, calculated with the program of Morton and Short (see [41]), whose $v$-span was 14 for both knots. Finally, an extensive check indeed found 4-braid representations of these knots, for example

$$
\sigma_{3}^{-1} \sigma_{2} \sigma_{3}^{3} \sigma_{2}^{2} \sigma_{1}^{2} \sigma_{2}^{2} \sigma_{1} \sigma_{3} \sigma_{2}^{2} \sigma_{1}^{2} \text { and } \sigma_{3}^{-1} \sigma_{2} \sigma_{3}^{2} \sigma_{2}^{2} \sigma_{1}^{2} \sigma_{2}^{2} \sigma_{1} \sigma_{3} \sigma_{2}^{2} \sigma_{1}^{2} \sigma_{2}
$$

Thus these two knots are braid positive, but have no positive minimal braid representation. (By the same remark on Cromwell's work as in example 1 these examples serve equally well also for homogeneous braid representations.)

Thus the question of whether a braid positive braid index $n$ knot has a positive minimal braid representation has a negative answer for $n=4$, and, taking connected sums of these knots with trefoils, also for $n>4$ (for this one needs to use e.g. the result of [15]). The only unsettled case remains $n=3$ (for $n=2$ the answer is positive by elementary arguments).

Question 2. If a knot $K$ has braid index 3 and is the closure of a positive braid, is it the closure of a positive 3-braid?

Unfortunately, we know from the work of Birman [6] and Murakami [42] that considering 3-braid knots just via their polynomials $P$ (and hence $V$ and $\Delta[3]$ ) and $Q$ will not suffice to give a positive answer to this question. 


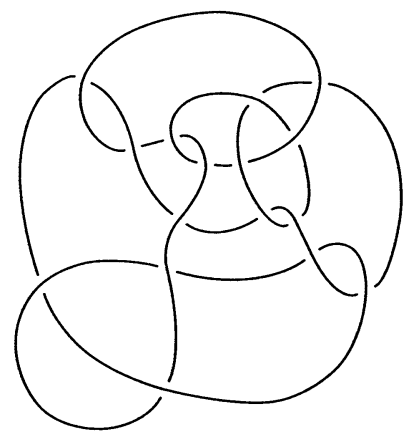

$16_{472381}$

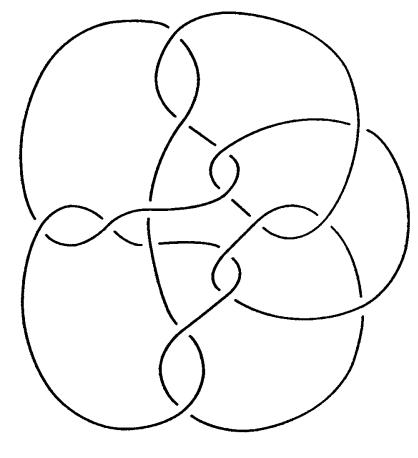

$16_{1223549}$

Figure 7. Two braid positive knots with no minimal positive braid representation.

In a similar way, taking iterated connected sums of the knots in example 7 and using the result of Cromwell [15, one obtains knots $K_{n}$ for which the difference between $b\left(K_{n}\right)$ and the positive braid index $b_{p}\left(K_{n}\right)$, the minimal strand number of a positive braid representation of $K_{n}$, becomes arbitrarily large. It would be interesting to find prime examples.

Question 3. Is there a sequence of prime braid positive knots $\left\{K_{n}\right\}$ for which $b_{p}\left(K_{n}\right)-b\left(K_{n}\right) \rightarrow \infty$ ?

3.2.3. Some examples on mutation. The next examples concern mutation. Mutation was introduced by Conway [13, and consists in building links which turn out very difficult to distinguish. Mutation replaces a tangle in a link diagram by its version rotated by $180^{\circ}$ around some axis (see [32]). The following example shows that for braid positive knots, mutation may not be visible in positive braid diagrams.

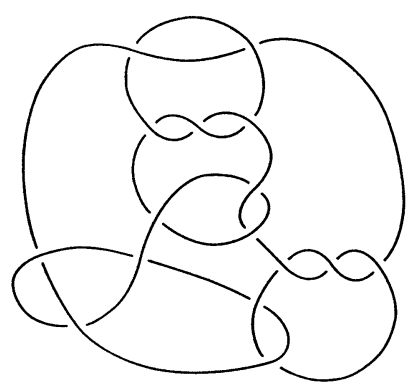

16473363

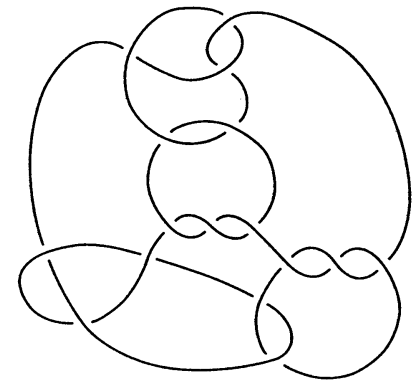

$16_{559253}$

Figure 8. Two braid positive mutants with no mutated positive braid diagrams.

To fix a bit of terminology, call a braid word reduced if it has no isolated generator. (This is not to be confused with the previous notion of irreducible, which is stronger.) 
Example 8. The knots $16_{473363}$ and $16_{559253}$ in figure 8 are iterated mutants. It turns out that both knots have a unique 16 crossing diagram which is a diagram of a closed positive braid (on 5 strands). The braids are

$$
\sigma_{1} \sigma_{2}^{2} \sigma_{3}^{3} \sigma_{1}^{2} \sigma_{4} \sigma_{2}^{3} \sigma_{3}^{3} \sigma_{4} \text { and } \sigma_{1} \sigma_{2}^{3} \sigma_{3}^{3} \sigma_{1} \sigma_{4} \sigma_{2}^{3} \sigma_{3}^{3} \sigma_{4} \text {. }
$$

We have already shown, in the proof of part 1 of theorem 3 (and its computational part, to follow in 93.3 .1$)$, that positive braids $\beta$ of genus 6 and $n \geq 7$ strands can be turned into braids with isolated generators by YB relations. If $\beta$ is itself reduced, then the YB relation giving an isolated generator is of the form $\sigma_{1} \sigma_{2} \sigma_{1} \rightarrow \sigma_{2} \sigma_{1} \sigma_{2}$ (or the same with $i$ replaced by $n-i, i=1,2$ ). Thus after reducing the isolated generator $\sigma_{1}$ (or $\left.\sigma_{n-1}\right)$ we obtain a braid with an edge generator $\left(\sigma_{1}\right.$ or $\left.\sigma_{n-1}\right)$ occurring in a square.

Moreover, it turns out that none of these knots occurs in a list of 17 crossing 6 -strand diagrams, which cannot be reduced in the above way (they all represent 14 and 15 crossing knots; see $\$ 3.3 .2$.

Thus, starting from a reduced braid representation of these knots of $\geq 6$ strands, by iteratedly applying YB relations and removing isolated (edge) generators, one must arrive at a 5-strand representation with an edge generator occurring in a square.

However, the second braid in (3) does not have this form. Thus it is the only reduced positive braid representation of $16_{559253}$.

Now the diagrams of the closures of the braids in (3) are easily seen not to be transformable by mutations (e.g., switch them to become alternating and calculate the Alexander polynomials - they differ). Also, since any other reduced positive braid diagram of $16_{473363}$ must have $>16$ crossings, it cannot be a mutated version of the 16 crossing positive braid diagram of $16_{559253}$, and the two knots have no mutated positive braid diagrams.

There is one further braid positive mutant in this group, $16_{488722}$, with 3 positive braid diagrams of 16 crossings, which are also not mutated versions of the one of $16_{559253}$.

(Also the braid closures of the braids in (3) are not mutants in the complement of their braid axis, as the skein polynomials of the 2-component links made up of braid closure and axis differ.)

Thus the problem of deciding whether positive braids are mutants is not solvable in the suggested way.

The next example shows that - perhaps even worse - the property of being braid positive is not preserved under mutation.

Example 9. The knots $15_{203528}$ and $15_{203969}$ in figure 9 belong to the same group of (iterated) mutants as $15_{203432}$ and $15_{203777}$ in figure 4, but their diagrams are positive braid diagrams, so they have positive braid representations.

3.3. Computational details. For the interested reader, here we make some supplementary remarks on how the above examples were obtained and checks were performed.

3.3.1. Theorem 3 First we address the proof of part 1) of theorem 3. We wanted to check that if $\beta$ is a positive braid (word) which is irreducible, i.e. inter alia modulo YB relations and cyclic permutations not transformable into a word with an isolated generator (i.e. letter appearing only once), then the subword $\beta_{1}$ of 


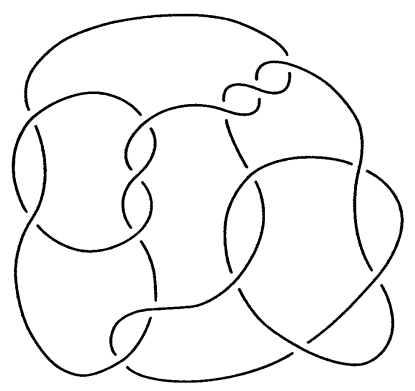

$15_{203528}$

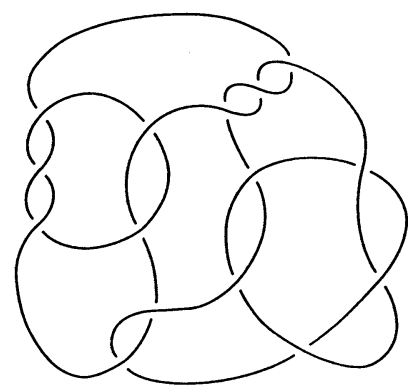

$15_{203969}$

Figure 9. These two mutants form with the knots in figure $4 \mathrm{a}$ group of iterated mutants, but, unlike the previous two knots, are braid positive.

' 1 ', ' 2 ' and ' 3 ' in $\beta$ has length at least 10. (We work with words of integers ' $i$ ' representing the corresponding generators $\sigma_{i}$, and in the sequel call these integers letters, rather than numbers.) We already know that the number of occurrences of ' 1 ' and ' 3 ' is at least 2 , and of ' 2 ' 'is at least 4 , and that the word $\beta_{1}$ must have length at least 9 . We want to show that no word of length 9 is possible.

This is done in 3 steps.

1) All words of letters ' 1 ', '2' and ' 3 ' of length 9 (representing candidates for $\beta_{1}$ ) are generated.

2) Irreducibility is tested. Irreducibility implies a number of conditions on the word $\beta_{1}$. To reduce the number of cases, we consider only words with maximal digit (= generator index) sum, and among them only those which are lexicographically minimal up to cyclic permutations. For such words the number of occurrences of ' 1 ', ' 2 ' and ' 3 ' is tested, and we also verify that the closure is not composite.

3) Connectedness test. Many braids $\beta$ can be discarded, as the non-connectedness of their closure can already be seen from their subwords $\beta_{1}$. We apply YB relations and cyclic permutations repeatedly to $\beta_{1}$, and eliminate squares (pairs of consecutive copies) of ' 1 ' and '2' (but not of ' 3 ', as there might be some letter ' 4 ' in $\beta$ between these two copies we have discarded building $\beta_{1}$ ). Whenever this procedure completely eliminates one of the letters ' 1 ' or ' 2 ', the closure is not a knot, and the braid (word) can be discarded.

These 3 checks already discard all possible words of ' 1 ', '2' and ' 3 ' of length 9 .

3.3.2. Proposition 1 and theorem 4 For examining 17 crossing 6 -braids, it suffices again to consider irreducible braids. To generate them, we use a similar method. We split the word (of letters ' 1 ' to ' 5 ') into its subwords $\beta_{1}$ of letters ' 1 ' to ' 3 ' and $\beta_{2}$ of letters ' 3 ' to ' 5 '. The words $\beta_{1}$ can be generated as above, and $\beta_{2}$ in a similar way with the following modifications/remarks.

- Replace $1 \rightarrow 5$ and $2 \rightarrow 4$. (This replacement means that, in composing $\beta_{1}$ and $\beta_{2}$ to $\beta$, we take $\beta$ 's maximal word representation modulo cyclic permutations, in which the letters ' 3 ' are weighted higher than ' 2 ' and ' 4 ', and they in turn are weighted higher than ' 1 ' and ' 5 '.) 
- The connectedness check can be applied to $\beta_{1}$ and $\beta_{2}$ in the same way as before (here, to determine connectedness, cyclic permutations are allowed also in $\left.\beta_{2}\right)$.

We can (up to flipping $2 \leftrightarrow 4$ and $1 \leftrightarrow 5$ ) assume that $\beta_{1}$ is not shorter than $\beta_{2}$. Then for a 17 crossing irreducible braid there are 3 possibilities, when keeping in mind the minimal number of occurrences of the letters (at least 4 for ' 2 ' and ' 4 ', at least 2 for ' 1 ', ' 3 ' and ' 5 ') and that $\left[\beta_{i}\right] \geq 10$ :

\begin{tabular}{c|c|c}
{$\left[\beta_{1}\right]$} & {$[\beta]_{3}$} & {$\left[\beta_{2}\right]$} \\
\hline 10 & 3 & 10 \\
10 & 4 & 11 \\
11 & 5 & 11
\end{tabular}

Given the words $\beta_{1,2}$, there is a canonical way of putting them together to obtain $\beta$ : the (sub)words $w_{1, i}$ of ' 1 ' and ' 2 ' between 2 occurrences of ' 3 ' in $\beta_{1}$ need to be composed with the subwords $w_{2, i}$ of ' 4 ' and ' 5 ' between the same occurrences of ' 3 ' in $\beta_{2}$. As the letters ' 1 ' and ' 2 ' commute with ' 4 ' and ' 5 ', by concatenating $w_{1, i}$ and $w_{2, i}$ we obtain the only relevant word.

The resulting diagrams can be checked for connectedness (note that the above connectedness tests were just partial) and the corresponding diagrams identified (they are about 1000). It turned out that only 6 knots occurred (two of 14 and four of 15 crossings), which all had braid positive minimal crossing diagrams.

To generate diagrams of $\leq 16$ crossings, a different approach was taken. From the alternating knot tables of [26] the fibred knots of the desired genus were selected by verifying the (degree and leading coefficient of their) Alexander polynomial. All flyped versions of their table diagrams were generated (flypes are the moves of [33]), and those knots were chosen which have an alternating braid diagram (the property of a diagram to be a braid diagram is not preserved by flypes, consider e.g. $7_{7}$ ). Then the table diagrams of these knots were switched to be positive (this commutes with flypes, so which alternating diagram of the knot is taken is no longer relevant), and the resulting knots were identified. These representations were found by examining the Jones polynomial.

A method similar to that of generating the ' 3 ' to ' 5 ' subwords was used in the quest for (almost positive 17 crossing) 4-braid representations of the knots in example 7. The word can be cyclically adjusted so that the negative generator will be first. Then the output of the 3-5 subword program (with ' 4 ' replaced by ' 2 ' and ' 5 ' replaced by ' 1 '; here it is relevant that no cyclic permutations are allowed to maximize the word) is appended to the negative crossing, and connectedness (of the closure) checked for the result. (Here the special meaning of the ' 3 ' in the connectedness check can be eliminated.)

\section{BRAID INDEX INEQUALITIES}

4.1. On a 4-braid criterion of Jones. In the famous paper [27], where Jones announced his fundamental discovery of a relation between $C^{*}$-algebras and Markov traces on braid groups, he also gave some results concerning applications of his new invariant to braids. Most of these, and many more, results have subsequently appeared with proof in his work-out [28]. One of these results (theorem 22 in [27]) 
was a formula relating the Jones polynomial $V$ and the Alexander polynomial $\Delta$ (normalized so that $\Delta(1)=1$ and $\Delta\left(t^{-1}\right)=\Delta(t)$ ) of 4 -braid knots.

Theorem 5 ([28, proposition 11.11]). If a knot $K$ is the closure of a 4-braid $\beta$ of exponent sum $[\beta]$, then

$$
\begin{aligned}
& t^{-[\beta]} V_{K}(t)+t^{[\beta]} V_{K}(1 / t) \\
&=\left(t^{-3 / 2}+t^{-1 / 2}+t^{1 / 2}+t^{3 / 2}\right)\left(t^{-[\beta] / 2}+t^{[\beta] / 2}\right) \\
&-\left(t^{-2}+t^{-1}+2+t+t^{2}\right) \Delta_{K}(t) .
\end{aligned}
$$

As a consequence of this theorem, in 27. Jones announced an obstruction to braid index 4 for knots (corollary 24), namely that the value $\Delta\left(e^{2 \pi i / 5}\right)$ for such knots must be of norm at most 6.5. This result did not appear with proof in [28], and my attempts to recover it failed. Finally, it turned out that the result, as stated there is in fact not correct, and there is a counterexample.

Example 10. The knot $13_{9221}$ of [26, shown in figure 10, has a (braid) diagram with 4 Seifert circles, and thus braid index at most 4 . (We have $\operatorname{span}_{v} P=6$, where $P$ is the HOMFLY polynomial 24, so by MFW [21 34] the braid index is indeed 4). However, its Alexander polynomial is $\Delta(t)=t^{-3}-10 t^{-2}+29 t^{-1}-39+29 t-10 t^{2}+t^{3}$, which at $t=e^{2 \pi i / 5}$ evaluates to $19 \sqrt{5}-49 \approx-6.5147084$.

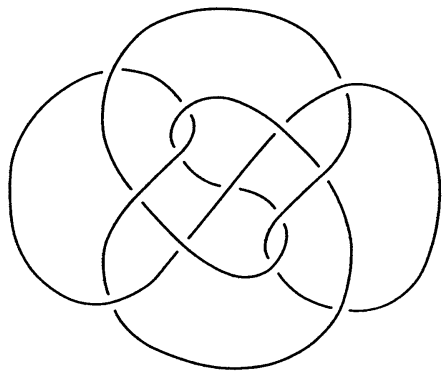

139221

FiguRe 10.

The value is still very close to 6.5 , and as this constant does not appear very natural, one suspects that it might have been obtained by rounding (possibly the difference of the estimates of (5) and (6) given below was taken erronously). However, there is some evidence that the bound cannot be fixed even just by a minor improvement. Instead we present a criterion with a larger, but definitely correct bound.

Proposition 2. If a knot $K$ has $\left|\Delta_{K}\left(e^{2 \pi i / 5}\right)\right|>6+2 \sqrt{5} \approx 10.472136$, then $K$ is not a closed 4-braid.

Proof. The formula (4) for $t=e^{2 \pi i / 5}$ simplifies to

$$
t^{-[\beta]} V_{K}(t)+t^{[\beta]} V_{K}(1 / t)=2 \cos [\beta] \pi / 5-\Delta_{K}(t),
$$

which, as $t$ has unit norm, and thus $V_{K}(1 / t)=\overline{V_{K}(t)}$, gives

$$
\Delta_{K}(t)=2 \cos [\beta] \pi / 5-2 \Re\left(t^{-[\beta]} V_{K}(t)\right) .
$$


We have

$$
|2 \cos [\beta] \pi / 5| \leq 2
$$

and from [28, proposition 14.6] also

$$
2 \Re\left(t^{-[\beta]} V_{K}(t)\right) \leq 2\left|V_{K}\left(e^{2 \pi i / 5}\right)\right| \leq 16 \cos ^{3}(\pi / 5)=\frac{(1+\sqrt{5})^{3}}{4}=4+2 \sqrt{5} .
$$

Putting (6) and (7) into (5) gives the result.

By remarking that $10 \nmid[\beta]$ (as $[\beta]$ is odd for a knot), one can slightly improve the upper estimate in (7), obtaining that

$$
\Delta_{K}\left(e^{2 \pi i / 5}\right) \leq \frac{9+5 \sqrt{5}}{2} \approx 10.09017
$$

for a 4-braid knot $K$.

The inequality (5) is clearly sharp, for $5 \mid[\beta]$ (it is not surprising that in the above example indeed $[\beta]=5$ ). Also, the second estimate in (7) is trivially sharp for links of braid index at most 4 (take the 4 component unlink), but the denseness result in [28, proposition 14.6] was sharpened in [54] to show that it remains true even if one restricts one's attention to knots, so that the only way to improve the bound in proposition 2 (resp. (8)) along these lines of argument is to improve the left inequality in (7), that is, to show that $t^{-[\beta]} V_{K}(t)$ is sufficiently far from the real line. This appears, however, unlikely as well, and thus a much better constant than the one given above can probably not be obtained.

The reason why Jones's 4-braid criterion never attracted particular attention is possibly that soon after it was announced, the (much more effective) MFW inequality was found. Indeed, for non-alternating prime knots of at most 14 crossings $13_{9221}$ was the only one which violated Jones's original (and insufficient) condition on $\Delta\left(e^{2 \pi i / 5}\right)$, and which had $\operatorname{span}_{v} P \leq 6$. An advantage of the (corrected) criterion involving $\Delta$ remains, however, that it is applicable also to very complicated knots because of the polynomial complexity of $\Delta$.

Finally, we mention that the above knot, $13_{9221}$, has another interesting property which is discussed in a joint paper with Mark Kidwell [30].

4.2. On the 2-cabled MFW inequality. In [40], Morton and Short introduced a way to circumvent MFW's failure to estimate sharply the braid index of a knot $K$ by applying the inequality on a 2-cable $K_{2}$ of $K$, a satellite around $K$ with a pattern intersecting each meridian disc of the solid torus twice and in the same direction. As this is a 2-braid, the satellite $K_{2}=K_{2, w}$ is uniquely determined by the writhe $w$ of this braid (the satellite is connected or disconnected depending on the parity of $w$ ). To obtain a braid representation of $K_{2, w}$ from a braid representation $\beta$ of $K$, a generator $\sigma_{i}$ in $\beta$ is replaced by $\sigma_{2 i} \sigma_{2 i-1} \sigma_{2 i+1} \sigma_{2 i}$, and the result is multiplied by $\sigma_{1}^{-2[\beta]+w}$. Thus $b\left(K_{2, w}\right) \leq 2 b(K)$, and, applying MFW on $K_{2, w}$, we obtain (for any $w \in \mathbb{Z})$

$$
b(K) \geq\left\lceil\frac{\operatorname{span}_{v} P_{K_{2, w}} / 2+1}{2}\right\rceil .
$$

This inequality completely determines the braid index of the Rolfsen knots, for which MFW itself failed. However, even this inequality sometimes fails, as the example below shows. As for this example the braid index to exclude is 4 (and hence the 3-braid conditions of [45] and [42] are not relevant), this gives an example 
of a knot on which all previously applied braid index criteria fail to estimate the braid index sharply.

Example 11. The knot $14_{45759}$ is depicted in figure11, Its $P$ polynomial is shown in table 1 and estimates the braid index to be at least 3 . This is however seen not to be exact from the $P$ polynomial of a 2-cable knot of $P$ shown below in table 10 estimating the braid index to be at least 4 . However, even this estimate is not exact. To see this, we use the fact that the knot is achiral, and we need to go a little behind the MFW inequality. This inequality was the consequence of the following two inequalities.

Theorem 6 ([34, 21]). If $K=\hat{\beta}, \beta \in B_{n}$, then $\min \operatorname{deg}_{v} P_{K} \geq[\beta]-n+1$ and $\max \operatorname{deg}_{v} P_{K} \leq[\beta]+n-1$.

If $14_{45759}$ were a closed 4 -braid $\beta$, then from the $P$ polynomial we see that only $[\beta]= \pm 1$ can occur, and indeed both values do because of achirality. Call these braids $\beta_{1,2}$. But then, taking the 2-cable of $\beta_{1,2}$, we obtain 8-braid representations of $K_{2}$ of different writhe. However, the fact that MFW is sharp for these 8-braids contradicts one of the two inequalities above. Thus the braid index of $14_{45759}$ is at least 5 .

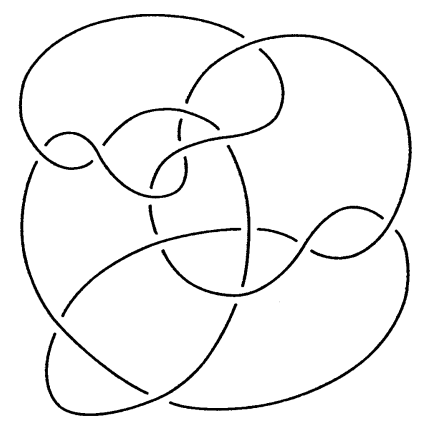

1445759

Figure 11.

4.3. The Jones conjecture. The example and the reasoning applied in the previous section can possibly be made more general.

An $n$-parallel $K_{\gamma}$ of $K$ is a satellite around $K$ of zero framing with pattern a closed $n$-string braid $\gamma$ in the solid torus given by the complement of its braid axis.

Lemma 1. If $\beta \in B_{n}$ is a braid representation for $K$, then $\langle\beta\rangle_{\gamma} \in B_{k n}$ is a braid representation for $K_{\gamma}, \gamma \in B_{k}$. Here

$$
\langle\beta\rangle_{\gamma}=\gamma \cdot\{\beta\}^{k} \cdot \Delta_{k}^{-2[\beta]},
$$

where $\{\beta\}^{k}$ is obtained from $\beta$ by the replacement

$$
\sigma_{i}^{\varepsilon} \longmapsto \prod_{n=1}^{2 k-1} \prod_{j=(i k-\min (n, 2 k-n)+1) / 2}^{(i k+\min (n, 2 k-n)-1) / 2} \sigma_{2 j}^{\varepsilon}, \quad \varepsilon= \pm 1,
$$


TABle 1 . The $P$ polynomial of $14_{45759}$ and a 2-cable knot of it.

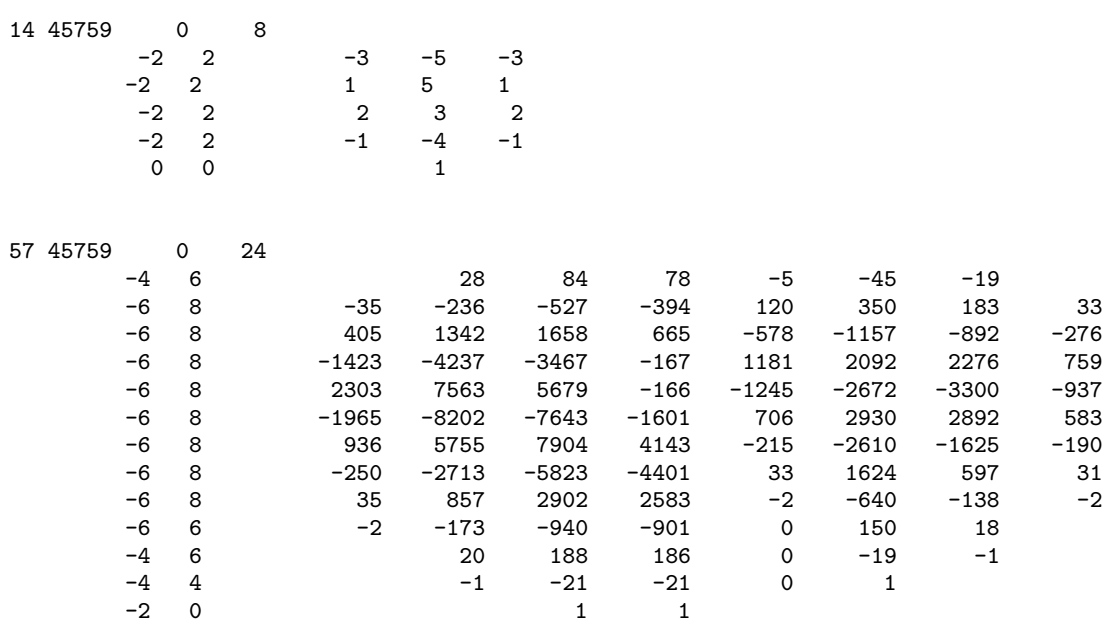

and

$$
\Delta_{k}=\prod_{j=1}^{k-1} \prod_{l=1}^{k-j} \sigma_{l}
$$

is the square root of the center generator of $B_{k}$ (the first and third factor on the right of (9) are meant w.r.t. the inclusion $B_{k} \hookrightarrow B_{n k}$ ).

Proof. This is a well-known and trivial fact (although seldom stated so explicitly). Taking the diagram $\hat{\beta}$ of $K$, we add $|[\beta]|$ kinks of sign $-\operatorname{sgn}[\beta]$, cable the diagram (under which $\beta$ is taken to $\{\beta\}^{k}$ ), and remove the kinks, obtaining $\Delta_{k}^{-2 \operatorname{sgn}[\beta]}$ for each kink.

Considering a (connected or disconnected) parallel $n$-cable knot (or link) $K_{n}$ of a knot $K$ (the choice of $\gamma \in B_{n}$ is no longer relevant), we can apply MFW to $K_{n}$ and use $b\left(K_{n}\right) \leq n b(K)$, thus obtaining an infinite series of inequalities (for any $n$ )

$$
b(K) \geq\left\lceil\left(\operatorname{span}_{v} P_{K_{n}} / 2+1\right) / n\right\rceil .
$$

The practical problem with these inequalities is that the calculation of $P_{K_{n}}$ is impossible for $n \geq 3$ and any, even moderately, interesting example $K$. Nevertheless, one can ask whether (10) can be made unsharp for smaller values of $n$, or even for all $n$.

It turns out that this problem is related to one of the still unsolved conjectures made by Jones shortly after his discoveries. It is as follows.

Conjecture 2 (Jones [28]). If $\beta, \beta^{\prime} \in B_{b(L)}$ and $\hat{\beta}=\hat{\beta}^{\prime}=L$, then $[\beta]=\left[\beta^{\prime}\right]$.

We include a brief historical review. The conjecture was first very implicitly mentioned in Jones's paper [28]. Later, some major publicity to it was given by Birman in her paper with Menasco [7], where it was proved (corollary, p. 267) that each link has at most finitely many writhes of minimal braid representations. However, this was proved previously by Morton in his paper [34, and also in [21], in a much less sophisticated way, not only for minimal, but for any arbitrary fixed strand number, and in stronger form, with very explicit lower and upper bounds to 
the writhe in terms of the degrees of the skein polynomial. In particular the Jones conjecture follows for links with sharp MFW inequality, or in fact for any link for which the braid index can be determined by applying MFW on some parallel cable, as in [41. Since this relationship was apparently not previously realized, it will be explained below.

Also, another statement of Birman needs correction. It is claimed that the Jones conjecture "is known to be true" for 4-braids "by the work of Jones" 28. However, at least in the case when a braid index 4 knot, as $10_{132}$, has the same skein polynomial as some knot of smaller braid index, Jones's formulas ([28, §8]) will certainly not be able to exclude multiple writhes of 4-braid representations, and already to classify when such duplications of the skein polynomial occur seems impossible. At least this was never (to the best of my knowledge) carried out by Jones. Jones himself only wrote that his formulas "lend some weight to the possibility" ([28, p. 357 , bottom]) that this conjecture is true. Thus the conjecture should be considered open in the 4-strand case (for 3-braids it was settled in [8]). In fact, a certain importance of 4-braids with regard to this conjecture will be established later.

An interesting special case of the Jones conjecture was addressed in a question raised independently by P. Johnson:

Question 4. Is there an achiral knot $K$ of even braid index?

The argument used in the previous example immediately shows that such a knot would be a counterexample to the Jones conjecture.

A generalization of our argument in $\$ 4.2$ yields the following explicit version of the Birman-Menasco result:

Theorem 7. For any knot $K$ and any $k \geq b(K)$ we have

$k-b(K)+1 \leq d_{k, K}=\#\left\{[\beta]: \beta \in B_{k}, \hat{\beta}=K\right\} \leq k-\max _{n, K_{n}}($ r.h.s. of (10) $)+1$.

Note that conjecture 2 is equivalent to $d_{k, K}=k-b(K)+1$ for any $K$ and $k \geq b(K)$.

Proof of theorem 7 . First observe that if $\beta, \beta^{\prime} \in B_{n}$, and $\gamma \in B_{k}$, then

$$
\left|\left[\langle\beta\rangle_{\gamma}\right]-\left[\left\langle\beta^{\prime}\right\rangle_{\gamma}\right]\right|=k\left|[\beta]-\left[\beta^{\prime}\right]\right| \text {. }
$$

To see this, use $\left[\Delta_{k}\right]=\frac{k(k-1)}{2}$ and $\left[\{\beta\}_{k}\right]=k^{2}[\beta]$. The result is straightforward from (9).

Now, it is immediate from the inequalities of theorem 6 that if $\beta, \beta^{\prime} \in B_{n}$ with $\hat{\beta}=\hat{\beta}^{\prime}=K$, then

$$
\frac{1}{2} \operatorname{span}_{v} P_{K}+1 \leq n-\frac{\left|[\beta]-\left[\beta^{\prime}\right]\right|}{2} .
$$

This already shows that the sharpness of the MFW inequality implies the truth of the Jones conjecture. However, we can get this relationship now in a more general version.

Applying (13) to $\langle\beta\rangle_{k}$ and $\left\langle\beta^{\prime}\right\rangle_{k}$, and using (12), we see that if $\beta, \beta^{\prime} \in B_{n}$ with $\hat{\beta}=\hat{\beta}^{\prime}=K$, and $\gamma \in B_{k}$, then

$$
\frac{1}{k}\left[\frac{1}{2} \operatorname{span}_{v} P_{K_{\gamma}}+1\right] \leq n-\frac{\left|[\beta]-\left[\beta^{\prime}\right]\right|}{2} .
$$


Let $D_{k, K}:=\left\{[\beta]: \beta \in B_{k}, \hat{\beta}=K\right\}$. As

$$
d_{k, K}=\# D_{k, K} \leq \max _{[\beta],\left[\beta^{\prime}\right] \in D_{k, K}} \frac{\left|[\beta]-\left[\beta^{\prime}\right]\right|}{2}+1,
$$

we get from (14) the second inequality in (11). The first inequality is trivial (take a minimal braid representation and stabilize in all possible ways).

The important consequence is the case $k=b(K)$ and $d_{k, K} \geq 2$ :

Corollary 2. If $K$ is a counterexample to conjecture 2, then 10 is unsharp for $K$ for any choice of $n$ and $K_{n}$.

This means that, provided we want to give a counterexample to conjecture 2 and even have found $\beta$ and $\beta^{\prime}$, we cannot prove their minimality using any of the inequalities (10). This shows why the quest for alternatives to MFW is worthwhile. There are such criteria, due to Jones [28], but (in particular, because MFW performs well very often) it is difficult to find examples where these criteria are more powerful than MFW. Although such examples exist (two are shown in the next section), they are very rare. Another handy criterion is Murakami's 3-braid formula [42, corollary 10.5]. This criterion is very effective - for example, it excludes (without a single failure!) from having braid index 3 all 916 non-alternating prime 15 crossing knots with MFW bound 3. (The candidates for exponent sums of 3-braids are taken from the skein polynomial.) As it applies only for 3-braid knots, at present it seems unrealistic to find a counterexample to the Jones conjecture in braid index $>4$. For the more optimistic readers, the corollary can also be taken as evidence for the conjecture.

In this situation I initiated a large computer experiment. I selected knots with MFW bound $\leq 3$ from the tables of [26], for which the Murakami test excludes braid index 3, but for which (7) is satisfied for (at least) two values of $[\beta]$ for $\beta \in B_{4}$ admitted by $P$ from the inequalities in theorem [6. Then I calculated $P$ of a 2-cable of these knots. This was already a non-trivial task. For knots for which I could find minimal crossing number diagrams with $\leq 5$ Seifert circles, I applied Vogel's algorithm [58]. The simplest (= lowest crossing number) braid representation obtained was 2 -cabled (a generator $\sigma_{i}$ replaced by $\sigma_{2 i} \sigma_{2 i-1} \sigma_{2 i+1} \sigma_{2 i}$ ) and processed by the program of [40, 41]. For the other knots the DT notation [19] of a 2-cable knot was generated from the DT notation of the knot (given in the tables of KnotScape), and the polynomial calculation program of KnotScape (a variation of the Millett-Ewing program) was used. This way (and with some assistance of Ken Millett for the hardest examples) I verified all prime knots up to 15 crossings, and many of the knots of 16 crossings, to which one of the Millett-Ewing or Morton-Short programs was applicable (for some knots both programs failed due to memory and time constraints). Although it did not give a counterexample to the Jones conjecture, this experiment found examples like the one of 4.2 ,

The following corollary summarizes for which classes of knots the sharpness of MFW, and hence the truth of the Jones conjecture, is known (see [21, 44]).

Corollary 3. The Jones conjecture is true for

- alternating fibred knots,

- rational knots, and

- positive braid knots with a full twist (i.e., closures of braids of the form $\Delta_{n}^{2} \alpha \in$ $B_{n}$ with $\alpha$ positive). 
4.4. The Jones polynomial at roots of unity. One of Jones's original criteria for the braid index came from the positivity of a scalar product on a $C^{*}$-algebra related to the values of his polynomial at primitive roots of unity.

Theorem 8 ([28, proposition 14.6]). If a knot $K$ has an $n$-braid representation and $k \geq 3$, then

$$
\left|V_{K}\left(e^{2 \pi i / k}\right)\right| \leq(2 \cos \pi / k)^{n-1} .
$$

This criterion turned out to be of less practical relevance than MFW, which is much more direct to apply and often more efficient. The fact that, however, the inequalities (15) can sometimes give better estimates, and thus need to be rehabilitated, is shown by the following example.

Example 12. The trefoil cable knot $K=13_{9465}=\left(3_{1}\right)_{2,7}$, shown in figure 12 has a $P$ polynomial giving the MFW bound 3 (this was noticed already in 21]). However, when considering its Jones polynomial (see table 2) and using (15) for $k=10$, we find that

$$
V_{K}\left(e^{\pi i / 5}\right)=-2-\frac{3+\sqrt{5}}{2} \sqrt{\frac{5-\sqrt{5}}{2}} i
$$

and thus

$$
\left|V_{K}\left(e^{\pi i / 5}\right)\right|^{2}=9+2 \sqrt{5} \approx 13.472136 \ldots,
$$

TABLE 2. The $P$ and $V$ polynomial of $13_{9465}$.

\begin{tabular}{rlllrrr}
139465 & \multicolumn{1}{c}{0} & 10 & & & \\
& 10 & 14 & & -11 & -15 & -5 \\
10 & 14 & & 40 & 35 & 5 \\
10 & 14 & & -57 & -28 & -1 \\
10 & 12 & & 36 & 9 & \\
10 & 12 & -10 & -1 & \\
10 & 10 & & 1 & &
\end{tabular}

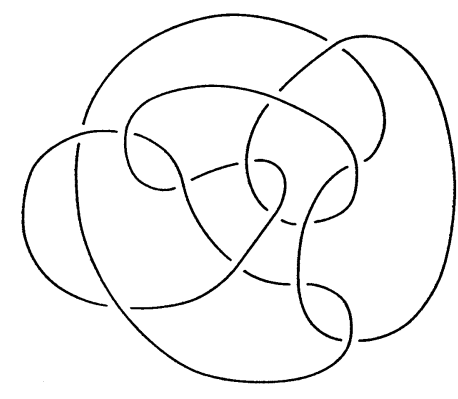

139465

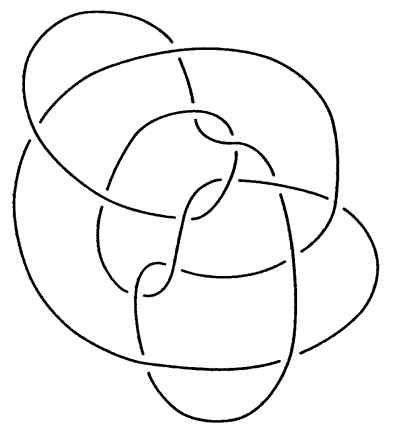

$14_{41800}$

FiguRE 12. 
which exceeds

$$
(2 \cos \pi / 10)^{4}=\frac{15+5 \sqrt{5}}{2} \approx 13.0901699 \ldots
$$

Thus $13_{9465}$ cannot be a 3-braid, and has braid index 4 (the diagram shows it to be a 4-braid). Similar examples (which are not closures of positive braids) are $14_{41800}$, $15_{138678}, 15_{141289}$ and $15_{251434}$.

The quest for such examples was motivated by the question (raised by Birman in problem 10.1 of [37]) on the realizability of the MFW bound among knots of given $P$ polynomial. This question is thus answered in the negative, first for MFW bound 3 , but examples for all higher values of the bound can be constructed by iteratedly taking (the polynomials of) connected sums of the above knot $13_{9465}$ with itself or with $5_{1}$ (or their mirror images). The cases remaining open are for MFW bound 1 and 2 .

Question 5. Are there $P$ polynomials of $v$-span $\leq 2$ other than those of the $(2, n)$ torus knots and links?

The problem for specific examples of polynomials is still difficult. Among the polynomials of the 5 Rolfsen knots with unsharp MFW (the two 9 crossing knots are addressed in problem 10.2 of [37]), the MFW bound is realizable for the polynomials of $10_{156}$ and $10_{132}$ (by $8_{16}$ and $5_{1}$ respectively, see [28, notes p. 386]), but the status of the remaining 3 polynomials is undecided. There is no prime knot of $\leq 16$ crossings duplicating the $P$ polynomial of $9_{42}$, and only one, $16_{730458}$, duplicating that of $9_{49}$. For the polynomial of $10_{150}$ there are two duplications in the tables, the knots being $13_{4977}$ and $13_{6718}$. However, for all these 3 duplicating knots the Murakami formula showed that they are not of braid index 3 (for 2 of them the 2-cabled MFW inequality even showed that the braid index is at least 5 ).

\section{The genus And HOMFLY POLYNOMIAL}

A final, and somewhat unrelated, collection of examples concerns two other conjectured relations of the degrees of the skein polynomial, this time related to genera.

5.1. Morton's conjecture. Shortly after the discovery of the skein polynomial, Morton [37] posed the question of whether for any link $L$,

$$
1-\chi(L) \geq \min \operatorname{deg}_{v} P(L),
$$

where $\chi(L)$ is the maximal Euler characteristic of a spanning surface for $L$ (if $L$ is a knot, then $1-\chi(L)$ is twice the genus of $L)$.

The motivation for this question was the fact that both sides of (16) are estimated below by $[\beta]-n(\beta)+1$ for any braid $\beta$ with $\hat{\beta}=L$. For the r.h.s. this was, as noted, proved by Morton himself, while for the l.h.s. it is a famous inequality of Bennequin [5], theorem 3].

Morton's conjecture resisted solution for a long time. (16) has been proved for homogeneous links [14] and 3-braid links [18], and has been verified computationally for prime knots with up to 16 crossings [57]. (Since both sides of (16) are additive under connected sum, it suffices to check prime links.) Also, further attempts have been made [16].

Here we settle Morton's conjecture negatively by means of counterexamples. 


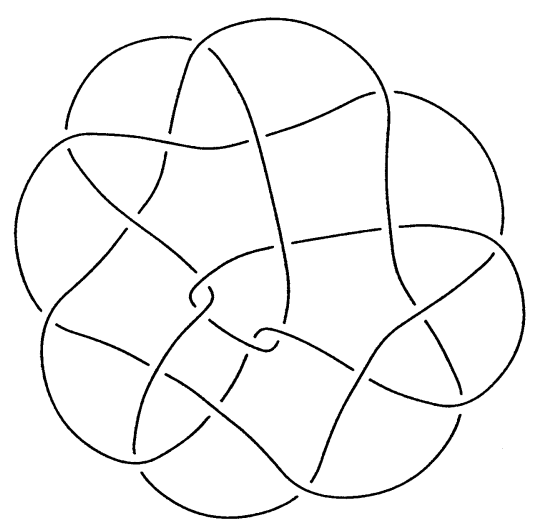

FiguRE 13. A counterexample to Morton's conjecture.

Example 13. Consider the knot $K$ in figure 13 It has the following 4-braid representation:

$$
\sigma_{1}^{2}\left(\sigma_{1} \sigma_{2} \sigma_{1}^{-1}\right)\left(\sigma_{2} \sigma_{3} \sigma_{2}^{-1}\right)\left(\sigma_{1} \sigma_{2} \sigma_{1}^{-1}\right)\left(\sigma_{2} \sigma_{3} \sigma_{2}^{-1}\right) \sigma_{3} \sigma_{1}\left(\sigma_{1} \sigma_{2} \sigma_{1}^{-1}\right)\left(\sigma_{1} \sigma_{2} \sigma_{3} \sigma_{2}^{-1} \sigma_{1}^{-1}\right) \sigma_{3} .
$$

From this representation it is evident that $K$ has a genus 4 surface obtained by connecting the 4 discs of the strands by the 11 bands indicated by the parenthesized subwords (see [50]). That this Seifert surface has minimal genus follows from Bennequin's inequality, since all the bands are 'positive'.

Thus $K$ has genus 4 . However, a calculation shows that $\min \operatorname{deg}_{v} P(K)=10$.

There are 7 further such examples, all given by 4-braids with 11 positive bands. For all 8 examples, KnotScape manages to reduce the diagrams to 21 crossings, while from the degree $\max \operatorname{deg}_{z} F=17$ of their Kauffman polynomial one concludes that their crossing number is at least 19. (They can be distinguished by the Kauffman polynomial, although the skein polynomials of several of them coincide.)

\subsection{Morton's canonical genus inequality.}

Example 14. A final example concerns another inequality of Morton, proved in 34]: $\max _{\operatorname{deg}_{z}} P_{K} \leq \tilde{g}(K)$. Here $\tilde{g}(K)$ is the canonical genus of a knot $K$, the minimal genus of the canonical Seifert surfaces of all its diagrams (see e.g. [56, 31]). Another obvious inequality is $g(K) \leq \tilde{g}(K)$. In a comparison of the 2 estimates for $\tilde{g}$, in 34 it was remarked that knots exist with $2 g<\max \operatorname{deg}_{z} P_{K}$. Since Morton's inequality is exact for very many knots (in particular all knots up to 12 crossings), in [38] I asked whether for some knot the opposite relation $2 g>\max \operatorname{deg}_{z} P_{K}$ can occur. Such examples indeed exist, and were found by implementing Gabai's method of disc decomposition [22] on canonical Seifert surfaces of special diagrams. See figure 14. This gives another example of knots with unsharp Morton inequality, after the ones found in [54].

\section{ACKNOWLEDGEMENT}

I would wish to thank Ken Millett for his help in doing a part of the calculations, and also V. Jones, H. Morton and K. Murasugi for helpful remarks and discussions. The referee's minor corrections are also acknowledged. 


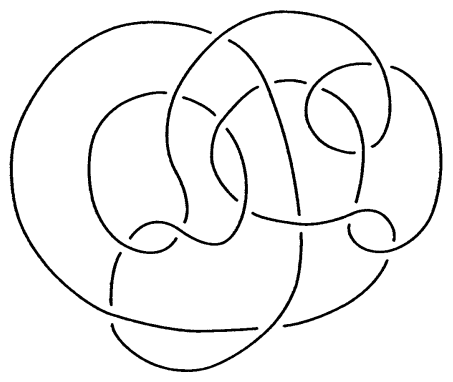

$15_{100154}$

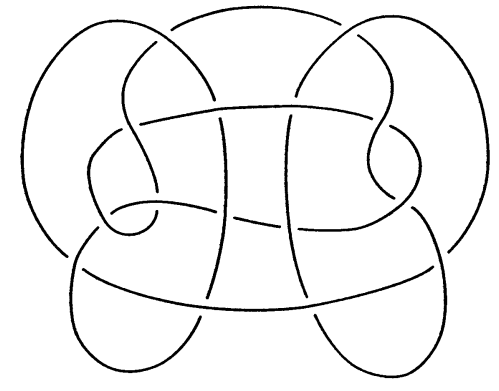

$15_{167945}$

Figure 14. Two knots for which $2 g>\max \operatorname{deg}_{z} P$. In both cases $\max \operatorname{deg}_{z} P=6$, while the canonical surfaces of the above diagrams are disc decomposable, and hence $g=4$. There are 10 further 15 crossing examples of this type.

\section{REFERENCES}

[1] C. C. Adams, Das Knotenbuch, Spektrum Akademischer Verlag, Berlin, 1995 (The knot book, W. H. Freeman and Co., New York, 1994). MR 94m:57007

[2] C. C. Adams et al., Almost alternating links, Topol. Appl. 46 (1992), 151-165. MR 93h:57005

[3] J. W. Alexander, Topological invariants of knots and links, Trans. Amer. Math. Soc. 30 (1928), 275-306.

[4] C. Aneziris, The mystery of knots. Computer programming for knot tabulation. Series on Knots and Everything 20, World Scientific, 1999.

[5] D. Bennequin, Entrelacements et équations de Pfaff, Soc. Math. de France, Astérisque 107108 (1983), 87-161. MR 86e:58070

[6] J. S. Birman, On the Jones polynomial of closed 3-braids, Invent. Math. 81 (1985), 287-294. MR 86m:57006

[7] J. S. Birman and W. W. Menasco, Studying knots via braids VI: A non-finiteness theorem, Pacific J. Math. 156 (1992), 265-285. MR 93m:57005

[8] _ Studying knots via braids III: Classifying knots which are closed 3 braids, Pacific J. Math. 161(1993), 25-113. MR 94i:57005

[9] J. S. Birman and R. F. Williams, Knotted periodic orbits in dynamical systems - I, Lorenz's equations, Topology 22(1) (1983), 47-82. MR 84k:58138

[10] R. D. Brandt, W. B. R. Lickorish and K. Millett, A polynomial invariant for unoriented knots and links, Invent. Math. 74 (1986), 563-573. MR 87m:57003

[11] J. van Buskirk, Positive links have positive Conway polynomial, Springer Lecture Notes in Math. 1144 (1983), 146-159. MR 87f:57007

[12] L. Carlitz, R. Scoville and T. Vaughan, Some arithmetic functions related to Fibonacci numbers, Fibonacci Quart. 11 (1973), 337-386. MR 48:10969

[13] J. H. Conway, An enumeration of knots and links, in "Computational problems in abstract algebra" (J. Leech, ed.), 329-358, Pergamon Press, 1969. MR 41:2661

[14] P. R. Cromwell, Homogeneous links, J. London Math. Soc. (series 2) 39 (1989), 535-552. MR 90f:57001

[15] _ Positive braids are visually prime, Proc. London Math. Soc. 67 (1993), 384-424. MR 95c:57008

[16] - A note on Morton's conjecture concerning the lowest degree of a 2-variable knot polynomial, Pacific J. Math. 160(2) (1993), 201-205. MR 94h:57008

[17] P. R. Cromwell and H. R. Morton, Positivity of knot polynomials on positive links, J. Knot Theory Ramifications 1 (1992), 203-206. MR 93i:57005

[18] O. T. Dasbach and B. Mangum, On McMullen's and other inequalities for the Thurston norm of link complements, Algebraic and Geometric Topology, 1 (2001), 321-347.

[19] C. H. Dowker and M. B. Thistlethwaite, Classification of knot projections, Topol. Appl. 16 (1983), 19-31. MR 85e:57003 
[20] Th. Fiedler, A small state sum for knots, Topology 32 (2) (1993), 281-294. MR 94c:57006

[21] J. Franks and R. F. Williams, Braids and the Jones polynomial, Trans. Amer. Math. Soc. 303 (1987), 97-108. MR 88k:57006

[22] D. Gabai, Foliations and genera of links, Topology 23 (1984), 381-394. MR 86h:57006

[23] F. Hirzebruch, Singularities and exotic spheres, Seminaire Bourbaki 10, Exp. No. 314, 13-32, Soc. Math. France, Paris, 1995. MR 99f:00042

[24] P. Freyd, J. Hoste, W. B. R. Lickorish, K. Millett, A. Ocneanu and D. Yetter, A new polynomial invariant of knots and links, Bull. Amer. Math. Soc. 12 (1985), 239-246. MR 86e:57007

[25] C. F. Ho, A polynomial invariant for knots and links - preliminary report, Abstracts Amer. Math. Soc. 6 (1985), 300.

[26] J. Hoste and M. Thistlethwaite, KnotScape, a knot polynomial calculation and table access program, available at http://www.math.utk.edu/ morwen.

[27] V. F. R. Jones, A polynomial invariant of knots and links via von Neumann algebras, Bull. Amer. Math. Soc. 12 (1985), 103-111. MR 86e:57006

[28] _ Hecke algebra representations of braid groups and link polynomials, Ann. of Math. 126 (1987), 335-388. MR 89c:46092

[29] T. Kanenobu, Relations between the Jones and Q polynomials of 2-bridge and 3-braid links, Math. Ann. 285 (1989), 115-124. MR 90i:57002

[30] M. Kidwell and A. Stoimenow, Examples Relating to the Crossing Number, Writhe, and Maximal Bridge Length of Knot Diagrams, Michigan Math. J. (to appear).

[31] M. Kobayashi and T. Kobayashi, On canonical genus and free genus of knot, J. Knot Theory Ramifications 5(1) (1996), 77-85. MR 97d:57008

[32] W. B. R. Lickorish and K. C. Millett, A polynomial invariant for oriented links, Topology 26 (1) (1987), 107-141. MR 88b:57012

[33] W. W. Menasco and M. B. Thistlethwaite, The Tait flyping conjecture, Bull. Amer. Math. Soc. 25 (2) (1991), 403-412. MR 92b:57017

[34] H. R. Morton, Seifert circles and knot polynomials, Math. Proc. Cambridge Philos. Soc. 99 (1986), 107-109. MR 87c:57006

[35] - The Burau matrix and Fiedler's invariant for a closed braid, Topol. Appl. 95(3) (1999), 251-256. MR 2001i:57025

[36] 260. MR 87c:57007

[37] (ed.), Problems, in "Braids", Santa Cruz, 1986 (J. S. Birman and A. L. Libgober, eds.), Contemp. Math. 78, 557-574. MR 89f:20003

[38] - (ed.), Problems, in the Proceedings of the International Conference on Knot Theory "Knots in Hellas, 98", Series on Knots and Everything 24, World Scientific, 2000, 547-559.

[39] H. R. Morton and E. A. El-Rifai, Algorithms for positive braids, Quart. J. Math. Oxford Ser. 2, 45(180) (1994), 479-497. MR 96b:20052

[40] H. R. Morton and H. B. Short, Calculating the 2-variable polynomial for knots presented as closed braids, J. Algorithms 11(1) (1990), 117-131. MR 91f:57004

[41] (1987), 267-278. MR 88f:57009

[42] J. Murakami, The Kauffman polynomial of links and representation theory, Osaka J. Math. 24(4) (1987), 745-758. MR 89c:57007

[43] K. Murasugi, On the braid index of alternating links, Trans. Amer. Math. Soc. 326 (1) (1991), 237-260. MR 91j:57009

[44] _ Jones polynomials and classical conjectures in knot theory, Topology 26 (1987), 187-194. MR 88m:57010

[45] _ On closed 3-braids, Memoirs Amer. Math. Soc. 151 (1974), American Mathematical Society, Providence, RI. MR 50:8496

[46] K. Murasugi and J. Przytycki, The skein polynomial of a planar star product of two links, Math. Proc. Cambridge Philos. Soc. 106(2) (1989), 273-276. MR 90f:57008

[47] T. Nakamura, Positive alternating links are positively alternating, J. Knot Theory Ramifications 9(1) (2000), 107-112. MR 2001a:57016

[48] D. Rolfsen, Knots and links, Publish or Perish, 1976. MR 58:24236]

[49] L. Rudolph, Nontrivial positive braids have positive signature, Topology 21(3) (1982), 325327. MR 83c:57009 
[50] — Braided surfaces and Seifert ribbons for closed braids, Comment. Math. Helv. 58 (1983), 1-37. MR 84j:57006

[51] N. J. A. Sloane, The On-Line Encyclopedia of Integer Sequences, accessible on the Internet address http://www.research.att.com/ njas/sequences.

[52] J. R. Stallings, Constructions of fibred knots and links, Algebraic and Geometric Topology (Stanford, CA, 1996), Proc. Sympos. Pure Math., vol. 32, part 2, Amer. Math. Soc., Providence, RI, 1978, pp. 55-60. MR 80e:57004

[53] A. Stoimenow, Positive knots, closed braids and the Jones polynomial, preprint math/9805078.

[54] Knots of genus two, preprint.

[55] - Generating functions, Fibonacci numbers, and rational knots, preprint.

[56] _ Knots of genus one, Proc. Amer. Math. Soc. 129(7) (2001), 2141-2156. MR 2002c: 57012

[57] _ Some inequalities between knot invariants, accepted by Internat. J. Math.

[58] P. Vogel, Representation of links by braids: A new algorithm, Comment. Math. Helv. 65 (1990), 104-113. MR 90k:57013

Max-Planck-Institut für Mathematik, Vivatsgasse 7, D-53111 Bonn, Germany

E-mail address: alex@mpim-bonn.mpg.de

Current address: Department of Mathematics, University of Toronto, Toronto, Canada M5S 3G3

E-mail address: stoimeno@math.toronto.edu

$U R L:$ http://www.math.toronto.edu/stoimeno 